\title{
Orthogonal Reverse Derivations on semiprime $\Gamma$-semirings
}

\author{
B. Venkateswarlu *, M. Murali Krishna Rao and Y. Adi Narayana
}

\begin{abstract}
In this paper, we introduce the notion of reverse derivation and orthogonal reverse derivations on $\Gamma$-semirings. Some characterizations of semi prime $\Gamma$-semirings are obtained by means of orthogonal reverse derivations. And also obtained necessary and sufficient conditions for two reverse derivations to be orthogonal.

Keywords: $\Gamma$-semirings; semi prime; derivations; orthogonal reverse derivations.

AMS Subject Classification (2010): Primary: 16Y60; Secondary: 06F35, 08A30, 03 G25.

${ }^{*}$ Corresponding author
\end{abstract}

\section{Introduction}

Semiring, the best algebraic structure, which is a common generalization of rings and distributive lattices was first introduced by American mathematician Vandiver [15] in 1934 but non trivial examples of semirings have appeared in the earlier studies on the theory of commutative ideals of rings by German mathematician Richard Dedekind in 19th century. Semiring is an universal algebra with two binary operations called addition and multiplication, where one of them distributive over the other, bounded distributive lattices are commutative semirings which are both additively idempotent and multiplicatively idempotent. A natural example of semiring is the set of all natural numbers under usual addition and multiplication of numbers. In particular, if $I$ is the unit interval on the real line then $(I, \max , \min )$ is a semiring in which 0 is the additive identity and 1 is the mutilative identity. The theory of rings and the theory of semigroups have considerable impact on the development of the theory of semirings. In structure, semirings lie between semigroups and rings. The study of rings shows that multiplicative structure of ring is independent of additive structure whereas in semiring multiplicative structure of semiring is not independent of additive structure of semiring. Additive and multiplicative structures of a semiring play an important role in determining the structure of a semiring. Semiring, as the basic algebraic structure, was used in the areas of theoretical computer science as well as in the solutions of graph theory and optimization theory and in particular for studying automata, coding theory and formal languages. Semiring theory has many applications in other branches. The notion of $\Gamma$-ring was introduced by Nobusawa [11] as a generalization of ring in 1964. Sen [13] introduced the notion of $\Gamma$-semigroup in 1981. The notion of Ternary algebraic system was introduced by Lehmer [5] in 1932, Lister [6] introduced ternary ring. Dutta \& Kar [3] introduced the notion of ternary semiring which is a generalization of ternary ring and semiring. In 1995, Murali Krishna Rao [7, 8] introduced the notion of $\Gamma$-semiring which is a generalization of $\Gamma$-ring, ring, ternary semiring and semiring. After the paper $[7,8]$ was published, many mathematicians obtained interesting results on $\Gamma$-semirings. Murali Krishna Rao and Venkteswarlu [9] introduced the unity element in $\Gamma$-semiring and studied properties of $\Gamma$-incline and field $\Gamma$-semiring.

Over the last few decades several authors have investigated the relationship between the commutativity of ring $R$ and the existence of certain specified derivations of $R$. The first result in this direction is due to Posner [12] in

Received : 18-01-2018, Accepted : 08-11-2018 
1957. In the year 1990, Bresar and Vukman [2] established that a prime ring must be a commutative if it admits a non zero left derivation. The notion of derivation of ring is useful for characterization of rings.

The notion of derivation of prime $\Gamma$-semirings was introduced by Javed et al. [4]. Suganthameena et al. [14] introduced the concept of orthogonal derivations on semirings. In this paper, we introduce the notion of reverse derivation and orthogonal reverse derivations on $\Gamma$-semirings. Some characterizations of semi prime $\Gamma$-semirings are obtained by means of orthogonal reverse derivations. And also obtained necessary and sufficient conditions for two reverse derivations to be orthogonal.

\section{Preliminaries}

In this section, we recall some important definitions which are necessary for this paper.

Definition 2.1. Let $(M,+)$ and $(\Gamma,+)$ be commutative semigroups. Then we call $M$ as a $\Gamma$-semiring if there exists a mapping $M \times \Gamma \times M \rightarrow M$ is written $(x, \alpha, y)$ as $x \alpha y$ such that it satisfying the following axioms for all $x, y, z \in M$ and $\alpha, \beta \in \Gamma$

(i) $x \alpha(y+z)=x \alpha y+x \alpha z$ (ii) $(x+y) \alpha z=x \alpha z+y \alpha z$

(iii) $x(\alpha+\beta) y=x \alpha y+x \beta y$ (iv) $x \alpha(y \beta z)=(x \alpha y) \beta z$.

Every semiring $R$ is a $\Gamma$-semiring with $\Gamma=R$ and ternary operation $x \gamma y$ as the usual semiring multiplication.

We illustrate the definition of $\Gamma$-semiring by the following example

Example 2.2. Let $S$ be a semiring and $M_{p, q}(S)$ denote the additive abelian semigroup of all $p \times q$ matrices with identity element whose entries are from $S$. Then $M_{p, q}(S)$ is a $\Gamma$-semiring with $\Gamma=M_{p, q}(S)$ ternary operation is defined by $x \alpha z=x\left(\alpha^{t}\right) z$ as the usual matrix multiplication, where $\alpha^{t}$ denotes the transpose of the matrix $\alpha$, for all $x, y$ and $\alpha \in M_{p, q}(S)$.

A $\Gamma$-semiring $M$ is said to have zero element if there exists an element $0 \in M$ such that $0+x=x=x+0$ and $0 \alpha x=x \alpha 0=0$, for all $x \in M, \alpha \in \Gamma$. A $\Gamma$-semiring $M$ is said to be commutative $\Gamma$-semiring if $x \alpha y=y \alpha x$, for all $x, y \in M$ and $\alpha \in \Gamma$. An element $a \in M$ is said to be an idempotent of $M$ if there exists $\alpha \in \Gamma$ such that $a=a \alpha a$ and $a+a=a$. Every element of $M$ is an idempotent of $M$ then $M$ is said to be idempotent $\Gamma$-semiring $M$. An element $1 \in M$ is said to be an unity if for each $x \in M$ there exists $\alpha \in \Gamma$ such that $x \alpha 1=1 \alpha x=x$.

A non-empty subset $A$ of $\Gamma$-semiring $M$ is called a $\Gamma$-subsemiring $M$ if $(A,+)$ is a subsemigroup of $(M,+)$ and $a \alpha b \in A$ for all $a, b \in A$ and $\alpha \in \Gamma$. A $\Gamma$-semiring $M$ is said to be prime if $a \Gamma M \Gamma b=0$ then $a=0$ or $b=0$, for all $a, b \in M$. A $\Gamma$-semiring $M$ is said to be 2-torsion free if $2 x=0$ then $x=0$, for all $x \in M$. A $\Gamma$-semirings $M$ is said to be semiprime if $a \Gamma M \Gamma a=0$ then $a=0$, for all $a \in M$. Every prime $\Gamma$-semiring is obviously semiprime. We write $[x, y]_{\alpha}=x \alpha y-y \alpha x$. For commutative $\Gamma$-semirings, $[x, y]_{\alpha}=0$, for every $x, y \in M$ and $\alpha \in \Gamma$. An additive mapping $d$ from $M$ into $M$ is called a derivation if $d(x \alpha y)=d(x) \alpha y+x \alpha d(y)$, for all $x, y \in M, \alpha \in \Gamma$.

\section{Orthogonal reverse derivation of $\Gamma$-semirings}

In this section, we introduce the notion of reverse derivation and orthogonal reverse derivations on $\Gamma$-semirings. Some characterizations of semi prime $\Gamma$-semirings are obtained by means of orthogonal reverse derivations. And also obtained necessary and sufficient conditions for two reverse derivations to be orthogonal.

Definition 3.1. Let $M$ be a $\Gamma$-semiring. An additive mapping $d$ from $M$ into $M$ is called a reverse derivation if $d(x \alpha y)=d(y) \alpha x+y \alpha d(x)$, for all $x, y \in M, \alpha \in \Gamma$.

If $M$ is commutative then both derivation and reverse derivation are the same.

Definition 3.2. Let $M$ be a $\Gamma$-semiring. An additive mapping $d$ from $M$ into $M$ is called a jordan derivation if $d(x \alpha x)=d(x) \alpha x+x \alpha d(x)$, for all $x \in M, \alpha \in \Gamma$.

In general, the reverse derivation is not a derivation but it is a jordan derivation.

Examples 3.3. 
(i) Let $S$ be a semiring and $d: S \rightarrow S$ be a reverse derivation. Consider $M=M_{1 \times 2}(S)$ and $\Gamma=\left\{n\left[\begin{array}{l}1 \\ 0\end{array}\right] \mid n \in Z^{+}\right\}$. Then $M$ is a $\Gamma$-semiring. Let $M_{1}=\{(x, y) \mid x, y \in S\} \subseteq M$.

Now define a mapping $D: M_{1} \rightarrow M_{1}$ by $D(x, y)=[d(x), d(y)]$, as $\left(x_{1}, y_{1}\right)\left(\begin{array}{c}n \cdot 1 \\ 0\end{array}\right)\left(x_{2}, y_{2}\right)=\left(x_{1} n x_{2}, y_{1} n y_{2}\right)$.

Therefore $D$ is a reverse derivation on $\Gamma$-semiring $M_{1}$.

(ii) Let $S$ be a semiring and $M=\left\{\left(\begin{array}{ll}x & y \\ 0 & 0\end{array}\right) \mid x, y \in S\right\}$ and $\Gamma=\left\{\left(\begin{array}{cc}n & 0 \\ 0 & 0\end{array}\right) \mid n\right.$ is an integer $\}$.

Then $M$ is a $\Gamma$-semiring. Now $d: M \rightarrow M$ is defined by $d\left(\begin{array}{ll}x & y \\ 0 & 0\end{array}\right)=\left(\begin{array}{ll}0 & y \\ 0 & 0\end{array}\right)$.

Therefore $d$ is a derivation but not reverse derivation.

(iii) Let $M$ be a semiring and $M=\left\{\left(\begin{array}{cccc}0 & x & y & z \\ 0 & 0 & 0 & y \\ 0 & 0 & 0 & -x \\ 0 & 0 & 0 & 0\end{array}\right) \mid x, y, z \in R\right\}$ and

$\Gamma=\left\{\left(\begin{array}{cccc}0 & 0 & 0 & 0 \\ 0 & n & 0 & 0 \\ 0 & 0 & n & 0 \\ 0 & 0 & 0 & n\end{array}\right) \mid n\right.$ is an integer $\}$. Then $M$ is a $\Gamma$-semiring.

Let $d: M \rightarrow M$ is defined by $d\left(\begin{array}{cccc}0 & x & y & z \\ 0 & 0 & 0 & y \\ 0 & 0 & 0 & -x \\ 0 & 0 & 0 & 0\end{array}\right)=\left(\begin{array}{cccc}0 & 0 & 0 & -z \\ 0 & 0 & 0 & y \\ 0 & 0 & 0 & -x \\ 0 & 0 & 0 & 0\end{array}\right)$.

Therefore $d$ is a reverse derivation but not derivation.

Definition 3.4. Let $d$ and $g$ be two reverse derivations on $\Gamma$-semiring $M$. If $d(x) \Gamma M \Gamma g(y)=0=g(x) \Gamma M \Gamma d(y)$, for all $x, y \in M$ then $d$ and $g$ are said to be orthogonal.

Note that a non-zero reverse derivation can't be orthogonal on it self.

Example 3.5. Let $d_{1}$ and $d_{2}$ be reverse derivations on $\Gamma_{1}$-semiring $M_{1}$ and $\Gamma_{2}$-semiring $M_{2}$ respectively. Consider $M=M_{1} \times M_{2}$ and $\Gamma=\Gamma_{1} \times \Gamma_{2}$. Now we define $(a, b)+(c, d)=(a+c, b+d)$ and $(a, b)(\alpha, \beta)(c, d)=(a \alpha c, b \beta d)$, for all $a, b \in M_{1}, c, d \in M_{2}, \alpha \in \Gamma_{1}, \beta \in \Gamma_{2}$. Then $M$ is a $\Gamma$-semiring.

Now define $d$ and $g$ on $M$ by $d(a, b)=\left(d_{1}(a), 0\right)$ and $g(a, b)=\left(0, d_{2}(b)\right)$. Then $d$ and $g$ are reverse derivations on $M$. And also it is clear that $d$ and $g$ are orthogonal reverse derivation on $M$.

Theorem 3.6. Let $M$ be a 2 -torsion free semiprime $\Gamma$-semiring. For a and $b$ are two elements of $M$, the following are equivalent

(i) $a \Gamma x \Gamma b=0$.

(ii) $b \Gamma x \Gamma a=0$.

(iii) $a \Gamma x \Gamma b+b \Gamma x \Gamma a=0$, for all $x \in M$.

If one of these conditions are fulfilled then $a \Gamma b=b \Gamma a=0$.

Proof. Let $a$ and $b$ be two elements of 2-torsion free semiprime $\Gamma$-semiring $M$.

(i) $\Rightarrow$ (ii) : Assume $a \Gamma x \Gamma b=0$, for all $x \in M$.

Pre and post multiplying by $b \Gamma x \Gamma$ and $\Gamma x \Gamma a$ then

$(b \Gamma x \Gamma a) \Gamma x \Gamma(b \Gamma x \Gamma a)=0$.

Therefore $b \Gamma x \Gamma a=0$, since $M$ is a semiprime, for all $x \in M$.

(ii) $\Rightarrow$ (i) : Suppose $b \Gamma x \Gamma a=0$, for all $x \in M$.

Pre and post multiplying by $a \Gamma x \Gamma$ and $\Gamma x \Gamma b$ then

$(a \Gamma x \Gamma b) \Gamma x \Gamma(a \Gamma x \Gamma b)=0$. Therefore $a \Gamma x \Gamma b=0$, for all $x \in M$. 
(ii) $\Rightarrow$ (iii) : Suppose $b \Gamma x \Gamma a=0$, for all $x \in M$. Then $a \Gamma x \Gamma b=0$, for all $x \in M$.

There fore $a \Gamma x \Gamma b+b \Gamma x \Gamma a=0$, for all $x \in M$.

(iii) $\Rightarrow$ (i): Suppose $a \Gamma x \Gamma b+b \Gamma x \Gamma a=0$, for all $x \in M \quad \cdots \quad(1)$

Pre multiplying by $b \Gamma x \Gamma$ then $b \Gamma x \Gamma a \Gamma x \Gamma b+b \Gamma x \Gamma b \Gamma x \Gamma a=0$.

Again pre multiplying by $a \Gamma x \Gamma$ then

$(a \Gamma x \Gamma b) \Gamma x \Gamma(a \Gamma x \Gamma b)+(a \Gamma x \Gamma b) \Gamma x \Gamma(b \Gamma x \Gamma a)=0 \quad \cdots \quad(2)$

Post multiplying (1) by $\Gamma x \Gamma a$ then $a \Gamma x \Gamma b \Gamma x \Gamma a+b \Gamma x \Gamma a \Gamma x \Gamma a=0$.

Again post multiplying by $\Gamma x \Gamma b$ then

$(a \Gamma x \Gamma b) \Gamma x \Gamma(a \Gamma x \Gamma b)+(b \Gamma x \Gamma a) \Gamma x \Gamma(a \Gamma x \Gamma b)=0 \quad \cdots \quad(3)$

Adding (2) and (3) then using (1), we get $2(a \Gamma x \Gamma b) \Gamma x \Gamma(a \Gamma x \Gamma b)=0$, for all $x \in M$.

Since $M$ is a 2-torsion free and semiprime then $a \Gamma x \Gamma b=0$, for all $x \in M$.

Let $a \Gamma x \Gamma b=0$, for all $x \in M$.

Pre and post multiplying by $b \Gamma$ and $\Gamma a$ respectively then $b \Gamma a \Gamma x \Gamma b \Gamma a=0$. Since $M$ is a semiprime, $b \Gamma a=0$.

Similarly, from $b \Gamma x \Gamma a=0$, we can show that $b \Gamma a=0$.

Theorem 3.7. Let $M$ be a 2-torsion free semi prime $\Gamma$-semiring. If additive mappings $d$ and $g$ of $M$ into itself satisfy $d(x) \Gamma M \Gamma g(x)=0$, for all $x \in M$ then $d(x) \Gamma M \Gamma g(y)=0$, for all $x, y \in M$.

Proof. Suppose $d(x) \Gamma m \Gamma g(x)=0$, for all $x, m \in M \cdots(1)$. Let $y \in M$.

$$
\begin{aligned}
0 & =d(x+y) \Gamma m \Gamma g(x+y) \\
& =d(x) \Gamma m \Gamma g(x)+d(x) \Gamma m \Gamma g(y)+d(y) \Gamma m \Gamma g(x)+d(y) \Gamma m \Gamma g(y) \\
& =d(x) \Gamma m \Gamma g(y)+d(y) \Gamma m \Gamma g(x) .
\end{aligned}
$$

Pre multiplying by $d(x) \Gamma m \Gamma g(y) \Gamma s \Gamma$, where $s \in M$,

$$
0=[d(x) \Gamma m \Gamma g(y)] \Gamma s \Gamma[d(x) \Gamma m \Gamma g(y)]+d(x) \Gamma m \Gamma[g(y) \Gamma s \Gamma d(y)] \Gamma m \Gamma g(x) \text {, for all } s \in M \cdots(2) .
$$

But from (1), $0=d(x) \Gamma s \Gamma g(x)$, for all $s \in M$

$$
\Rightarrow 0=g(x) \Gamma s \Gamma d(x) \text {, by Theorem 3.6, for all } x, s \in M \text {. }
$$

Now from (2), $0=d(x) \Gamma m \Gamma g(y) \Gamma s \Gamma d(x) \Gamma m \Gamma g(y)$

$$
\Rightarrow \quad 0=d(x) \Gamma m \Gamma g(y) \text {, since } M \text { is a semiprime, for all } x, y, m \in M \text {. }
$$

Hence the theorem.

Theorem 3.8. Let $M$ be a 2-torsion free semiprime $\Gamma$-semiring, $d$ and $g$ be reverse derivations of $M$ into itself. Then $d$ and $g$ are orthogonal if and only if $d(x) \alpha g(y)+g(x) \alpha d(y)=0$, for all $x, y \in M, \alpha \in \Gamma$.

Proof. Suppose $d(x) \alpha g(y)+g(x) \alpha d(y)=0$, for all $x, y \in M, \alpha \in \Gamma$.

Replace $y$ by $x \beta y$, where $\beta \in \Gamma$, then we obtain

$$
\begin{aligned}
0 & =d(x) \alpha g(x \beta y)+g(x) \alpha d(x \beta y) \\
\Rightarrow \quad 0 & =d(x) \alpha[g(y) \beta x+y \beta g(x)]+g(x) \alpha[d(y) \beta x+y \beta d(x)] \\
\Rightarrow \quad 0 & =d(x) \alpha g(y) \beta x+d(x) \alpha y \beta g(x)+g(x) \alpha d(y) \beta x+g(x) \alpha y \beta d(x) . \\
\Rightarrow \quad 0 & =\{d(x) \alpha g(y)+g(x) \alpha d(y)\} \beta x+d(x) \alpha y \beta g(x)+g(x) \alpha y \beta d(x) . \\
\Rightarrow \quad 0 & =0+d(x) \alpha y \beta g(x)+g(x) \alpha y \beta d(x) .
\end{aligned}
$$

By Theorem 3.6, $d(x) \alpha y \beta g(x)=0=g(x) \alpha y \beta d(x)$, for all $x, y \in M, \alpha, \beta \in M$.

By Theorem 3.7, $d(x) \alpha y \beta g(z)=0=g(x) \alpha y \beta d(z)$, for all $x, y, z \in M, \alpha, \beta \in M$.

Thus $d$ and $g$ are orthogonal.

Conversely assume that $d$ and $g$ are orthogonal. Then $d(x) \Gamma m \Gamma g(y)=g(x) \Gamma m \Gamma d(y)=0$, for all $x, y, m \in M$. By Theorem 3.6, $d(x) \Gamma g(y)=0=g(x) \Gamma d(y) \Rightarrow d(x) \Gamma g(y)+g(x) \Gamma d(y)=0$, for all $x, y \in M$.

Hence the theorem. 
Remark 3.9. Suppose $d$ and $g$ are reverse derivations of $\Gamma$-semiring on $M$. Then for $x, y \in M, \alpha \in \Gamma$,

$$
\text { (i) } \begin{aligned}
d g(x \alpha y) & =d(g(x \alpha y))=d(g(y) \alpha x+y \alpha g(x))=d(g(y) \alpha x)+d(y \alpha g(x)) \\
& =d(x) \alpha g(y)+x \alpha d g(y)+d g(x) \alpha y+g(x) \alpha d(y) \\
\text { ii) } g d(x \alpha y) & =g(d(x \alpha y))=g(d(y) \alpha x+y \alpha d(x))=g(d(y) \alpha x)+g(y \alpha d(x)) \\
& =g(x) \alpha d(y)+x \alpha g d(y)+g d(x) \alpha y+d(x) \alpha g(y) .
\end{aligned}
$$

Theorem 3.10. Let $M$ be a 2 -torsion free semi prime $\Gamma$-semiring. Suppose $d$ and $g$ are reverse derivations of $M$ into $M$. Then $d$ and $g$ are orthogonal if and only if $d g=0$.

Proof. Let $M$ be a 2-torsion free semiprime $\Gamma$-semiring and $d$ and $g$ be two reverse derivations of $M$ into $M$. Suppose $d g=0$. Let $x, y \in M, \alpha \in \Gamma$. From Remark 3.9 and $d g=0$, we get $d g(x \alpha y)=d(x) \alpha g(y)+g(x) \alpha d(y)=0$. By Theorem 3.8, $d$ and $g$ are orthogonal.

Conversely suppose that $d$ and $g$ are orthogonal. Then

$$
\begin{aligned}
0 & =d(x) \alpha y \beta g(z), \text { for all } x, y, z \in M, \alpha, \beta \in \Gamma . \\
\Rightarrow 0 & =d[d(x) \alpha y \beta g(z)] \\
\Rightarrow 0 & =d g(z) \beta y \alpha d(x)+g(z) \beta d(y) \alpha d(x)+y \beta g(z) \alpha d[d(x)] .
\end{aligned}
$$

Since $d$ and $g$ are orthogonal, then second and third summands are zero.

Therefore, we obtain, $d g(z) \beta y \alpha d(x)=0$, for all $x, y, z \in M, \alpha, \beta \in \Gamma$.

Now $x$ replace by $g(z)$ then $d g(z) \beta y \alpha d g(z)=0$. Since $M$ is a semi prime, we get $d g(z)=0$, for all $z \in M$.

Hence $d g=0$.

Corollary 3.11. Let $M$ be a 2 -torsion free semi prime $\Gamma$-semiring. Suppose $d$ and $g$ are reverse derivations of $M$ into $M$. Then $d$ and $g$ are orthogonal if and only if $g d=0$.

Theorem 3.12. Let $M$ be a 2 -torsion free semiprime $\Gamma$-semiring and $d$ and $g$ be reverse derivations of $M$ into $M$. Then $d$ and $g$ are orthogonal if and only if $d g+g d=0$.

Proof. Let $M$ be a 2-torsion free semiprime $\Gamma$-semiring and $d$ and $g$ be reverse derivations of $M$ into $M$.

Let $x, y \in M, \alpha \in \Gamma$.

$$
\begin{aligned}
\text { Suppose } 0 & =d g+g d . \\
\Rightarrow 0 & =(d g+g d)(x \alpha y)=d g(x \alpha y)+g d(x \alpha y) \\
\Rightarrow 0 & =2 d(x) \alpha g(y)+2 g(x) \alpha d(y) \\
\Rightarrow 0 & =d(x) \alpha g(y)+g(x) \alpha d(y), \text { since } M \text { is a } 2-\text { torsion free. }
\end{aligned}
$$

By Theorem 3.8, $d$ and $g$ are orthogonal. The proof of the converse follows from Theorem 3.10 and Corollary 3.11

Theorem 3.13. Let $M$ be a 2 -torsion free semiprime $\Gamma$-semiring. Suppose $d$ and $g$ are reverse derivations of $M$ into $M$. Then $d$ and $g$ are orthogonal if and only if $d g$ is a derivation.

Proof. Let $M$ be a 2-torsion free semiprime $\Gamma$-semiring. Suppose $d$ and $g$ are reverse derivations of $M$ into $M$. Suppose that $d$ and $g$ are orthogonal. Let $x, y \in M$ and $\alpha \in \Gamma$. From Remark 3.9 and Theorem 3.8, we get $d g(x \alpha y)=d g(x) \alpha y+x \alpha d g(y)$. Hence $d g$ is a derivation.

Conversely suppose that $d g$ is a derivation. Let $x, y \in M$ and $\alpha \in \Gamma$. Now $d g(x \alpha y)=d g(x) \alpha y+x \alpha d g(y)$.

Therefore Remark 3.9 and comparing then we get $0=d(x) \alpha g(y)+g(x) \alpha g(y)$.

Therefore, by Theorem 3.8, $d$ and $g$ orthogonal.

Corollary 3.14. Let $M$ be a 2 -torsion free semiprime $\Gamma$-semiring. Suppose that $d$ and $g$ are reverse derivations of $M$ into itself. Then $d$ and $g$ are orthogonal if and only if $g d$ is a derivation.

Corollary 3.15. Let $M$ be 2 -torsion free semiprime $\Gamma$-semiring. Suppose $d$ and $g$ are reverse derivations on $M$. Then the following are equivalent.

(i). $d$ and $g$ are orthogonal 
(ii). $d g=0$

(iii). $g d=0$

(iv). $d g+g d=0$

(v). $d g$ is derivation

(vi). gd is derivation

The proof of the following corollary from Theorem 3.6 and Corollary 3.15.

Corollary 3.16. Let $d$ and $g$ be reverse derivations on 2 -torsion free semiprime $\Gamma$-semiring $M$. Suppose $d$ and $g$ are orthogonal on $M$. Then either $d=0$ or $g=0$.

Theorem 3.17. Let $M$ be a 2 -torsion free semiprime $\Gamma$-semiring. If $d$ is a reverse derivation of $M$ into $M$ such that $d^{2}$ is a derivation then $d=0$.

Proof. Suppose $d^{2}$ is a derivations 2 -torsion free semiprime $\Gamma$-semiring $M$ and $x, y \in M, \alpha \in \Gamma$.

$$
\begin{aligned}
\text { Now } d^{2}(x \alpha y) & =d(d(x \alpha y)) \\
\Rightarrow d^{2}(x) \alpha y+x \alpha d^{2}(y) & =d[d(y) \alpha y+y \alpha d(x)] \\
& =d(x) \alpha d(y)+x \alpha d^{2}(y)+d^{2}(x) \alpha y+d(x) \alpha d(y) \\
\Rightarrow 2 d(x) \alpha d(y) & =0 \\
\Rightarrow d(x) \alpha d(y) & =0, \text { since } M \text { is a 2-torsion free, for all } x, y \in M, \alpha \in \Gamma \cdots(1)
\end{aligned}
$$

Replace $x$ by $z \beta x$ in (1), $z \in M, \beta \in \Gamma$, we get

$$
\begin{aligned}
0 & =d(z \beta x) \alpha d(y)=[d(x) \beta z+x \beta d(z)] \alpha d(y) \\
\Rightarrow 0 & =(d(x) \beta z) \alpha d(y)+x \beta(d(z) \alpha d(y)) \\
\Rightarrow 0 & =(d(x) \beta z) \alpha d(y), \quad \text { from }(1) \quad \cdots(2)
\end{aligned}
$$

Replace $y$ by $x+y$ in (2), we get,

$$
\begin{aligned}
0 & =(d(x) \beta z) \alpha d(x+y)=d(x) \beta z \alpha d(x)+d(x) \beta z \alpha d(y) \\
\Rightarrow 0 & =d(x) \beta z \alpha d(x), \text { from (2), for all } x, z \in M, \alpha, \beta \in \Gamma . \\
\Rightarrow 0 & =d(x), \text { since } M \text { is a semiprime, for all } x \in M .
\end{aligned}
$$

This completes the proof.

Theorem 3.18. Let $M$ be a 2 -torsion free semiprime $\Gamma$-semiring. Suppose $d$ and $g$ are reverse derivations on $M$. If $d^{2}=g^{2}$ then the following are holds

(i). $(d+g)$ and $(d-g)$ are orthogonal.

(ii). either $d=-g$ or $d=g$.

Proof. Let $M$ be a $2-$ torsion free semiprime $\Gamma$-semiring. Suppose $d$ and $g$ are reverse derivations on $M$ and $d^{2}=g^{2}$.

(i).

$$
\begin{gathered}
{[(d+g)(d-g)+(d-g)(d+g)](x)=(d+g)(d(x)-g(x))+(d-g)(d(x)+g(x))} \\
=d(d(x))-d g(x)+g d(x)-g(g(x))+d(d(x)+d(g(x)-g d(x)-g(g(x))=0 .
\end{gathered}
$$

By Theorem 3.12, $d+g$ and $d-g$ are orthogonal.

(ii). From (i), $d+g$ and $d-g$ are orthogonal. By Theorem 3.16, $d+g=0$ or $d-g=0$.

Then $d=-g$ or $d=g$.

The proof of the following corollary from Theorems 3.8 and 3.18 .

Corollary 3.19. Let $M$ be a 2-torsion free semiprime $\Gamma$-semiring and $d$ and $g$ are reverse derivations on $M$. If $d(x) \alpha d(x)=$ $g(x) \alpha g(x)$, for all $x \in M$ then $d+g$ and $d-g$ are orthogonal. 


\section{References}

[1] Allen, P.J., A fundamental theorem of homomorphism for semirings, Proc. Amer. Math. Soc., 21 (1969), 412-416.

[2] Bresar, M. and Vukman, J., On the left derivation and related mappings, Proc. Amer. Math. Soc., 10 (1990), 7-16.

[3] Dutta, T.K. and Kar, S., On regular ternary semirings, Advances in Algebra, Proceedings of the ICM Satellite Conference in Algebra and Related Topics, World Scientific, (2003) 343-355.

[4] Javed, M.A., Aslam, M. and Hussain, M., On derivations of prime $\Gamma$-semirings, Southeast Asian Bull. of Math., 37 (2013), 859-865.

[5] Lehmer, H., A ternary analogue of abelian groups, Amer. J. of Math., 59 (1932), 329-338.

[6] Lister, W.G., Ternary rings, Tran. of Amer. Math. Soc., 154 (1971), 37-55.

[7] Murali Krishna Rao, M., $\Gamma$-semirings-I, Southeast Asian Bull. of Math., 19(1) (1995), 49-54.

[8] Murali Krishna Rao, M., Г-semirings-II, Southeast Asian Bull. of Math., 21 (1997), 281-287.

[9] Murali Krishna Rao, M. and Venkateswarlu, B., Regular $\Gamma$-semirings and field $\Gamma$-semirings, Novi Sad J. of Math., 45 (2) (2015), 155-171.

[10] Neumann, V., On regular rings, Proc. Nat. Acad. Sci., 22 (1936), 707-13.

[11] Nobusawa, N., On a generalization of the ring theory, Osaka. J. Math., 1 (1964), 81 - 89.

[12] Posner, E.C., Derivations in prime rings, Proc. Amer. Math. Soc., 8 (1957), 1093-1100.

[13] Sen, M.K., On $\Gamma$-semigroup, Proc. of International Conference of algebra and its application, (1981), Decker Publicaiton, New York, 301-308.

[14] Suganthameena, N. and Chandramouleeswaran, M., Orthogonal derivations on semirings, Int. J. Of Cont. Math. Sci., 9 (2014), 645-651.

[15] Vandiver, H.S., Note on a simple type of algebra in which the cancellation law of addition does not hold, Bull. Amer. Math., 40 (1934), 914-921.

\section{Affiliations}

\section{B. VENKATESWARLU}

AdDrESS: Department of Mathematics, GST, GITAM University, Doddaballapur- 562 163, Banguluru North, Karnatka, India.

E-MAIL: bvlmaths@gmail.com

ORCID ID: 0000-0003-3669-350X

M. MURALI KRISHNA RAO

ADDRESS: Department of Mathematics, GIT, GITAM University, Visakhapatnam- 530 045, A.P., India.

E-MAIL: mmarapureddy@gmail.com

ORCID ID: 0000-0003-4798-1259

Y. ADI NARAYANA

ADDRESS: Department of Mathematics, NRI IT, Agiripalli- 521 212, Vijayawada, A.P., India.

E-MAIL: yan_msc@yahoo.com

ORCID ID: 0000-0002-8836-3732 